Edited by H.-D. Esperer, M.D.

\title{
Biosignal Analysis
}

\section{Basics}

- Biosignal Analysis:

http://www.cs.ucy.ac/project/mi/biosign.htm

\section{Data Compression}

- ECG Data Compression Techniques: http://hcs.harvard.edu/ /weber/HomePage/Papers/ECGCompression/

\section{SA-ECG}

- Body Surface Potential Mapping: http://wwww.heartweb.org/1196/ep0006.htm

- SA-ECG: Detection of Abnormalieties (Autocorrelation plotting) http://php.iupui.edu/ eamuneva/seniot_design/ee492.htm

\section{Wavelet Analysis}

- SA-ECG: Dectection of Time and Frequency Abnormalities (Wavelet Analysis) http://www.geocities.com/CollegePark/Library/1765REF13.html

- ECG Analysis Using Wavelets: http://www.wavelet.org/wavelet/digest_05_05.04.html

\section{Pattern Recognition}

- ECG Signal Analysis (Pattern Recognition, Hidden Markov Models) http://cs.utu.fi/staff/antti.koski/abs.html

- ECG-Signal Analysis: Fuzzy Clustering http://funsan.biomed.mcgill.ca/ funnell/embc95_cd/texts/682.htm

\section{Nonlinear Analysis}

- ECG-Signal Analysis: Nonlinear Analysis http://circhp.epfl.ch/ schimmin/uni/beleg/body.html

- The Human ECG: Nonlinear versus stochastic aspects http://citesser.nj.nec.com/130774.html

\section{Clinical Source}

- Clinical Capnography http://shr.hama-med.ac.jp/iscaic18/AbstratSymposium/Jgoldman.html

Note: Although, the above links have been tested by us and proved to be accessible at the time of printing, some links may have been moved or may have exspired. 